\title{
ESTUDO COMPARATIVO ENTRE AS SOLUÇÕES DE MANITOL, PICOSSULFATO DE SÓdIO E FOSFATO MONOBÁSICO E DIBÁSICO DE SÓDIO NO PREPARO DE CÓLON PARA COLONOSCOPIA ${ }^{1}$
}

\author{
COMPARISON OF COLON CLEANSING METHODS IN \\ PREPARATION FOR COLONOSCOPY.
}

\author{
Paulo Miki Jr² \\ José Joaquim Ribeiro da Rocha ${ }^{3}$ \\ Francisco Aprilli ${ }^{3}$ \\ Omar Féres $^{3}$
}

\section{RESUMO}

Atualmente, a colonoscopia é o exame de primeira escolha na avaliação das doenças do cólon, tendo também papel fundamental na abordagem terapêutica de determinado grupo de patologias. O preparo intestinal é etapa obrigatória para a realização das colonoscopias eletivas, e a qualidade deste encontra-se diretamente relacionada ao sucesso do procedimento, seja este diagnóstico ou terapêutico. Foram comparadas três soluções para limpeza anterógrada do cólon em pacientes adultos, submetidos à colonoscopia em regime ambulatorial. Sessenta pacientes foram distribuidos em três grupos de vinte indivíduos. Cada um dos grupos realizou o preparo do cólon com uma das três soluções estudadas: manitol a $10 \%(\mathrm{MN})$, picossulfato sódico (PS) e fosfato monobásico e dibásico de sódio $(\mathrm{NaP})$. O sabor, a tolerância, os efeitos colaterais, os custos e a qualidade de limpeza do preparo foram avaliados. Parâmetros clínicos como frequência cardíaca e pressão arterial sistêmica também foram estudados, além das variações dos eletrólitos (sódio, potássio, cálcio e fósforo) que foram dosados antes e após o preparo intestinal. Os pacientes estudados apresentaram resultados semelhantes em relação aos efeitos colaterais. As variações relativas à freqüência cardíaca, pressão arterial e hematócrito, ainda que indicando contração no espaço intravascular, não cursaram com maiores repercussões do ponto de vista clínico. O sabor ruim da solução de $\mathrm{NaP}$ não chegou a comprometer a aceitação desta pe- los pacientes. As soluções de NaP e MN proporcionaram resultados superiores tanto em qualidade de limpeza colônica, como em relação aos custos, quando comparadas à solução de PS. Quando comparados os três grupos do estudo, todos os eletrólitos avaliados apresentaram diferenças significativas, sendo a hiperfosfatemia dos pacientes preparados com a solução de $\mathrm{NaP}$, a mais importante delas. Nenhuma dessas, no entanto, apresentou problemas de ordem clínica. Disponível em URL: http://www.scielo.br/acb

Descritores - colonoscopia; preparo de cólon; fosfato de sódio; Manitol

\begin{abstract}
Currently, colonoscopy has been a first choice exam to evaluate colon disease. This procedure also has had essencial role in the therapeutic approach of certain groups of pathologies. Colonic cleansing is a necessary and previous stage to do elective colonoscopy. The quality of the colon preparation has been found related directly to the sucess of procedure. Three solutions were compared as colonic cleansing agents in adult patients submitted to colonoscopy at ambulatory regimen. Sixty patients were randomized and then divided into three groups of twenty persons. Each one of these groups was submitted to a colonic cleansing with one of these studied solutions: $10 \%$ mannitol solution, sodium picosulphate and sodium phosphate. Taste, tolerance, associated side effects and
\end{abstract}

\footnotetext{
Trabalho realizado no Departamento de Cirurgia e Anaotmia - FMRP - USP

Pós-graduando e Médico Contratado do Departamento de Cirurgia e Anatomia- FMRP - USP

Docente do Departamento de Cirurgia e Anatomia - FMRP - USP
} 
quality of cleansing preparation were evaluated. Clinic parameters like standing pulse and blood pressure besides the assessment of sodium, potassium, calcium and phophate were also studied and compared with measurements taken before and after bow el preparation of each one of patients. The groups presented similar results when related to side effects. Electrolyte alterations were verified in these three studied groups without clinical signs. The variations related to cardiac frequency, blood pressure and hematocrit, though showing contraction of the intravascular volume, did not attend with clinic effects. The bad taste of sodium phosphate solution did not compromise its tolerance. The sodium phosphate and $10 \%$ mannitol solutions provide superior results in quality of colonic cleansing and as much as costs when compared to sodium picosulphate solution. When these three groups were compared all evaluated electrolytes presented significant differences and hyperphosphatemia of patients prepared with sodium phosphate solution was the most important of then. None of these differences was related to clinic effects.

Key Words - Bowel preparation; Sodium phosphate; Mannitol; Sodium picosulphate.

\section{INTRODUÇÃO}

Nos últimos anos a colonoscopia tem se firmado como o procedimento de escolha, tanto para o diagnóstico como para o tratamento das mais variadas lesões colônicas, tendo suplantado o enema opaco em nosso meio. No entanto, para que se obtenha sucesso, seja nos exames diagnósticos ou terapêuticos, há a necessidade de um preparo colônico satisfatório quer no sentido da limpeza da luz do cólon, como também na aceitação à ingesta do produto e possíveis efeitos colaterais associados aos métodos de preparo empregados.

No que diz respeito à limpeza mecânica do cólon existem duas possibilidades: a via anterógrada, que utiliza o sentido natural do trânsito intestinal e a retrógrada, feita basicamente através de lavagens por via retal.

Ao longo dos anos, a maioria dos autores vêm dando preferência ao preparo anterógrado do cólon, pela qualidade de limpeza obtida e pelo conforto do paciente. Para esta finalidade, várias soluções de preparo foram utilizadas nos últimos trinta anos, sendo as mais conhecidas: a solução salina ${ }^{1}$, o manitol ${ }^{2}$, o polietilenoglicol ${ }^{3}$, o picossulfato de sódio ${ }^{4}$ a solução de fosfato de sódio ${ }^{5}$.

Diante de poucas referências comparando cientificamente o uso dessas diversas soluções para o preparo de cólon, desenvolvemos este estudo, que tem como objetivo avaliar três soluções para o preparo de cólon para colonoscopia. Compara o preparo intestinal com o manitol a $10 \%(\mathrm{MN})$ àqueles utilizando as soluções orais de sulfato monobásico e dibásico de sódio (NaP) e de picossulfato de sódio (PS), objetivando apontar qual das soluções estudados proporciona melhores resultados em relação à qualidade de limpeza colônica, as alterações hidroeletrolíticas e suas possíveis repercussões clínicas, efeitos colaterais, sabor, tolerância e custos operacionais relacionados a cada um dos processos de limpeza em questão.

\section{MÉTODOS}

Sessenta pacientes submetidos a exames endoscópicos ambulatoriais foram divididos de modo sequencial em três grupos de vinte pacientes cada.

Como critérios de exclusão foram considerados: infarto agudo do miocárdio, insuficiência cardíaca, insuficiência renal, ascite, sinais sugestivos de suboclusão intestinal, colectomia prévia, acidente vascular cerebral além de quadros abdominais agudos.

Todos os pacientes receberam a mesma orientação dietética, a qual preconizava a ausência de resíduos na ante-véspera do exame e dieta líquida exclusiva, além de $10 \mathrm{mg}$ de laxante de contato (picossulfato de sódio) no dia anterior à colonoscopia. Foi estimulado o aumento do consumo de líquidos ao longo do preparo, apenas proibindo-se o consumo de leite, seus derivados e bebidas contendo corantes escuros.

O grupo de pacientes selecionados para o preparo com a solução de $\mathrm{MN}$ iniciou o mesmo com quatro horas de antecedência ao horário do exame, tendo que consumir $1.000 \mathrm{ml}$ da solução em um período de 1 hora. Os que foram orientados a realizar o preparo com a solução de $\mathrm{NaP}$ (fosfato de sódio monobásico $\mathrm{H} 2 \mathrm{O}-$ 16gr; fosfato de sodio dibásico $7 \mathrm{H} 2 \mathrm{O}$ - 6gr; veículo q.s.p. $-100 \mathrm{ml}$ ), o fizeram consumindo os $130 \mathrm{ml}$ da referida solução em um máximo de 15 minutos, três horas antes dos exames. Aqueles destinados ao preparo colônico com a solução de PS (dois saches, cada um deles contendo: picossulfato sódico:10mg; óxido de magnésio: $3,5 \mathrm{~g}$; ácido cítrico: $12 \mathrm{~g}$; dióxido de silício: $0,2 \mathrm{~g}$; bicarbonato de potássio: $0,5 \mathrm{mg}$; sacarina sódica: $60 \mathrm{mg}$ e aromatizante sabor laranja: $60 \mathrm{mg}$ ) foram orientados a consumir o produto conforme orientações do próprio fabricante (um sache diluído em $200 \mathrm{ml}$ de água), às 8 e 16 horas da véspera do exame. Todos os pacientes do estudo foram encorajados a consumir volumes elevados de líquidos ao longo do período de preparo.

Pouco antes do início dos exames, todos os pacientes responderam a um questionário padrão, dan- 
do informações a respeito da tolerância, efeitos colaterais, sabor e outros possíveis aspectos relacionados ao preparo.

A qualidade de cada preparo foi avaliada a partir dos dados referidos pelo examinador em um relatório. Foram considerados como referência os parâmetros estabelecidos por Vanner et al. (1990) $)^{5}$ e graduados em excelente (ausência ou pequeno volume de líquido claro no interior do cólon), bom (grande volume de líquido claro, sem resíduos), regular (resíduos fecais com possibilidade de aspiração e/ou lavagem seguida de aspiração) e ruim (resíduos fecais semi-sólidos, impossíveis de lavagem/aspiração adequados).

Dosagens séricas de sódio, potássio, cálcio, fósforo, hemoglobina e hematócrito foram realizadas em dois momentos: 24 horas antes do início do preparo e quando da punção venosa, no início do procedimento endoscópico. Parâmetros clínicos como pressão arterial sistêmica e suas variações posturais, frequência cardíaca e saturação de oxigênio no sangue periférico, foram sistematicamente monitorizadas.

A análise estatística para comparação das alterações de cada grupo foi feita com o teste de Wilcoxon. Para as avaliações entre os três grupos empregou-se o teste de Kruskal-Wallis nas comparações globais, sendo complementado pelo teste de Miller. Na análise dos efeitos colaterais foi utilizado o teste do Qui-quadrado ( $\mathrm{x} 2$ ).

Para todos os testes o valor de $5 \%(\mathrm{p}<0,05)$ ou inferior foi considerado como sendo significativo.

\section{RESULTADOS}

Todos os sessenta pacientes foram capazes de completar o regime de preparo proposto, embora tenha havido melhor aceitação da solução de PS, seguida pelo $\mathrm{NaP}$ e, por último, a solução de MN. Apenas a diferença entre a de melhor aceitação (PS) e a de MN foi avaliada como tendo significado estatístico $(\mathrm{p}<0,05)$.

O PS também mostrou-se superior em relação ao $\mathrm{MN}$ e ao NaP quanto ao sabor, sendo esta última solução significativamente inferior às duas primeiras na referida avaliação.

Em relação à qualidade do preparo, $78,33 \%$ dos pacientes estudados apresentaram preparos de excelente ou boa qualidade e em apenas $3,33 \%$ deles, esta foi avaliada como sendo ruim. O grupo que fez uso do NaP obteve $95 \%$ dos índices entre excelente e bom preparo. Já os pacientes preparados com o $\mathrm{MN}$, atingiram $90 \%$ dos casos e o grupo do PS, apenas $50 \%$ de qualidade adequada de preparo. A diferença supracitada é significativa do ponto de vista estatístico quando comparados os resultados obtidos entre o grupo do PS e as outras duas soluções estudadas $(\mathrm{p}<0,05)$.

Não houve diferença estatisticamente significativa entre os três grupos estudados no que se refere a efeitos colaterais, chegando-se ao valor de $86,66 \%$ dos pacientes apenas referindo queixas leves durante o período de preparo do cólon.

As variações da pressão arterial sistêmica antes e após o preparo do cólon foram avaliadas tanto com os pacientes em posição ortostática, como em decúbito dorsal, mostrando nítida tendência a queda dos valores de pressão sistólica em ambas as posições e em todos os grupos, porém sem significado estatístico.

No que se refere à freqüência cardíaca, os três grupos de pacientes do estudo em questão apresentaram aumento da mesma ao término do preparo, não havendo diferença estatística entre eles.

Os grupos de MN e PS apresentaram elevação significatica do hematócrito $(\mathrm{p}<0,05)$ quando comparados os dados de antes e após o preparo, tendo-se observado exatamente o oposto com o grupo preparado com o NaP. Índices de significância também foram encontrados quando comparados os resultados dos dois primeiros grupos ao do NaP.

Em relação às variações dos eletrólitos, o sódio apresentou queda após o preparo com o PS $(p<0,05)$ e elevação no $\mathrm{MN}(\mathrm{p}<0,05)$ e no $\mathrm{NaP}(\mathrm{p}>0,05)$. Comparados os três grupos, há significância estatística apenas na diferença encontrada entre o PS e as outras duas soluções. Já em relação ao potássio, houve diminuição dos valores após o preparo no grupo do PS $(p>0,05)$ e do $\mathrm{NaP}(p<0,05)$, e elevação no grupo do $\mathrm{MN}(\mathrm{p}>0,05)$. As diferenças entre os três grupos são significativas apenas quando comparados o $\mathrm{NaP}$ com o MN.

No que diz respeito ao fósforo, não houve significado estatístico nas alterações verificadas nos valores obtidos nas duas dosagens, tanto no grupo preparado com a solução de $\mathrm{MN}$, quanto naquele em que a solução utilizada foi o PS $(\mathrm{p}>0,05)$. Porém, quando considerados os resultados observados no grupo de paciente, que fez uso da solução de $\mathrm{NaP}$, verifica-se acentuado aumento do fósforo sérico ao término do preparo $(\mathrm{p}<0,05)$. Comparados os três grupos, encontra-se resultado significativo entre as diferenças do grupo do $\mathrm{NaP}$ e as duas outras soluções do estudo.

Considerando-se o cálcio sérico, verificamos elevação na dosagem realizada após o preparo no grupo da solução de PS $(p<0,05)$ e queda nos referidos valores quando avaliadas as soluções de $\mathrm{NaP}(\mathrm{p}>0,05)$ e $\mathrm{MN}$ $(p>0,05)$. A diferença entre os três grupos volta a ser 
significativa, porém apenas quando confrontados os valores referentes às soluções de $\mathrm{NaP}$ e PS.

Em relação aos custos relacionados a cada uma das soluções estudadas, a de NaP mostrou-se a mais acessível, seguida da solução de MN e, por fim, do PS.

\section{DISCUSSÃO}

Os dados apresentados nos permitem constatar uma clara superioridade da solução de PS, quando avaliamos a tolerância e o sabor das soluções utilizadas neste estudo. Podemos atribuir tal resultado à combinação do paladar agradável e ao pequeno volume da referida solução como sendo os fatores preponderantes para este achado, pois não encontramos esta mesma associação nas outras duas soluções, seja pelo maior volume no caso do $\mathrm{MN}$ ou pelo sabor ruim da solução de NaP. Mesmo assim, indiferentemente à tolerância ou ao sabor das soluções, todos os pacientes foram capazes de completar os diferentes preparos propostos.

Os efeitos adversos associados às soluções de preparo de cólon vêm apresentando freqüência e conseqüências cada vez menos expressivas desde que foi abolido o uso da solução salina. Com o surgimento de soluções para preparo anterógrado do cólon, temos observado efeitos colaterais cada vez menos intensos e, geralmente restritos a cólicas, náuseas, vômitos e meteorismo abdominal ${ }^{6}$. O presente estudo demonstrou que em $28 \%$ dos pacientes não foram observados efeitos colaterais. Pacientes que informaram apenas um efeito adverso somaram um total de $58 \%$ do total. Esses dois grupos, juntos, chegam à cifra de $86 \%$ de todos os pacientes apenas referindo queixas pouco significativas do ponto de vista clínico. Em 1,5\% do total dos pacientes foram constatadas alterações clínicas de maior relevância, sempre associadas à desidratação.

No que diz respeito à qualidade do preparo do cólon para a colonoscopia, existe consenso por parte dos autores em afirmar que o sucesso e a segurança do exame encontram-se diretamente relacionados à limpeza obtida, independentemente da opção feita em relação ao método utilizado ${ }^{7,8}$.

No nosso estudo, os resultados apontam $95 \%$ de qualidade de limpeza entre excelente e boa no grupo de pacientes preparados com a solução de $\mathrm{NaP}, 90 \%$ no grupo do $\mathrm{MN}$ e $50 \%$ nos pacientes preparados com o PS. Há diferença significativa entre os dois primeiros grupos ( $\mathrm{NaP}$ e $\mathrm{MN}$ ) e este último. A principal razão para esta diferença foi a presença de fezes aderentes no cólon direito de boa parte dos pacientes preparados com a solução de PS.
Com o avanço das pesquisas e, consequentemente das soluções de preparo, as diferenças encontradas na qualidade do preparo têm mostrado resultados bastante aproximados, fato que torna dados associados à tolerância e custos operacionais, relevantes ou mesmo inerentes a um bom método de preparo do cólon ${ }^{9,10}$. O presente estudo mostra uma avaliação recente de custos associados aos três diferentes produtos analisados, considerando para tal, apenas os valores obtidos junto a estabelecimentos comerciais os quais, por sua vez, tinham como referência dados fornecidos pela Brasindice. Foi observada uma pequena diferença de preço entre as soluções de $\mathrm{MN}$ e $\mathrm{NaP}$, sendo esta última mais acessível. Já em relação à solução de PS, houve uma diferença considerável em comparação aos outros dois produtos avaliados, uma vez que o custo final desta chega a ser aproximadamente $50 \%$ e $36 \%$ superior às soluções de $\mathrm{NaP}$ e $\mathrm{MN}$ respectivamente.

A grande maioria dos estudos atuais têm demonstrado graus variados de desidratação, associados às diferentes soluções de preparo anterógrado do cólon. Em nosso trabalho, os dados relativos à freqüência cardíaca, pressão arterial sistêmica e hematócrito indicaram que houve uma redução no espaço intravascular dos pacientes. Tal alteração, em paralelismo à literatura, foi avaliada como sendo pouco representativa do ponto de vista clínico, sem nenhuma repercussão sobre a segurança dos preparos intestinais estudados.

Além da permanente busca por alternativas que proporcionassem limpeza intestinal adequada, as alterações eletrolíticas provocadas pelos diferentes preparos estudados, assim como suas possíveis implicações, tornaram-se preocupação freqüente no sentido de se conseguir soluções que causassem anormalidades de menores repercussões nos níveis dos eletrólitos séricos ${ }^{11,12}$. A partir dos resultados deste estudo pôdese constatar que nos pacientes preparados com o $\mathrm{NaP}$ e com o MN, houve aumento nos valores de sódio ao término do preparo intestinal, sendo esta elevação significativa apenas no grupo do $\mathrm{MN}$. Em relação ao PS, verificou-se hiponatremia significativa quando da segunda dosagem. Comparados os três grupos, foram significativas as diferenças entre o grupo do PS em relação ao $\mathrm{MN}$ e $\mathrm{NaP}$, o mesmo não ocorrendo entre os dois últimos.

No que diz respeito ao potássio, foi encontrada hipopotassemia significativa no grupo do $\mathrm{NaP}$ e não significativa com o PS. A elevação anotada no grupo de $\mathrm{MN}$, contrária à maioria dos relatos da literatura, também não atingiu valores estatisticamente significativos. Ao confrontarmos os três grupos, houve significado estatístico apenas nas diferenças encontradas entre os grupos de $\mathrm{NaP}$ e $\mathrm{MN}$. 
Vários relatos da literatura têm referido elevações acentuadas nas dosagens de fósforo sérico nos pacientes cujo preparo é realizado com a solução de $\mathrm{NaP}$, incluindo um caso isolado de óbito associado a tal alteração. A já esperada hiperfosfatemia em 100\% dos pacientes do grupo do $\mathrm{NaP}$, foi a alteração mais importante nos estudos relacionados aos eletrólitos e também a que maior índice de significado estatístico obteve. O grupo de pacientes preparado com o $\mathrm{MN}$ também mostrou elevação do fósforo ao término deste, porém sem significado estatístico associado. Praticamente não houve alteração nos valores do grupo do PS. As diferenças anotadas entre o grupo do $\mathrm{NaP}$ e os demais foram estatisticamente significativas. Em nenhum paciente do grupo do $\mathrm{NaP}$ foram observadas alterações clínicas de maior relevância.

Em referência ao cálcio, assim como na literatura, houve queda dos valores séricos ao final da limpeza do cólon quando foram utilizadas as soluções de $\mathrm{NaP}$ e $\mathrm{MN}$, sem índices significativos, o que foi constatado na elevação do referido eletrólito com a solução de PS. Entre os três grupos, apesar de haver significado estatístico na diferença entre eles, tal fato apenas se deu entre os grupos de $\mathrm{NaP}$ e PS, sem que efeitos adversos fossem verificados.

\section{CONCLUSÃO}

Com base nos resultados obtidos no presente estudo, conclui-se que as soluções de $\mathrm{NaP}$ e $\mathrm{MN}$ proporcionaram resultados de limpeza colônica semelhantes e significativamente superiores à solução de PS. Alterações hidroeletrolíticas estiveram associadas às três soluções de preparo estudadas, não tendo havido repercussões do ponto de vista clínico, no sentido de prejudicar a segurança na realização de cada preparo. Não houve diferença entre os três grupos de pacientes estudados no que diz respeito aos efeitos colaterais apresentados.

\section{REFERÊNCIAS}

1. Hewitt J, Rigby J, Reeve J, Cox AG. Whole gut irrigation in preparation for large bowel surgery. Lancet 1973; 2: 337-40.

2. Champault G, Patel JC. Apropos of the communication by L.F. Hollender (12 october 1977): Our experience with washout in colon surgery. Colon preparation; usefulness of digestive irrigation with oral absorption of 10 percent Mannitol. Chirurgie 1977; 103: 998-9.

3. Davis GR, Santa Ana CA, Morawski SG, Fordtran JS. Development of a lavage solution associated with minimal water and electrolyte absorption or secretion. Gastroenterology 1980; 78: 991-5.

4. De Lacey G, Benson M, Wilkins R, Spencer J, Cramer B. Routine colonic lavage is unnecessary for double-contrast barium enema in outpatients. Br Med J 1982; 1021-2.

5. Vanner SJ, Macdonald MD, Paterson WG, Prentice RSA, DaCosta LR, Beck IT. A randomized prospective trial comparing oral sodium phosphate with standard polyethylene glycol-based lavage solution (Golytely) in the preparation of patients for colonoscopy. Am J Gastroenterol 1990; 85(4): 422-7.

6. Habr-Gama A, Hahas SC, Calache JE, Sousa Jr AHS, Alves PA, Araujo SEA, Bringel RWA, Marques CFS. Preparo intestinal para colonoscopia: solução de manitol vs solução de fosfato de sódio. Estudo prospectivo e randomizado. Rev Bras Coloproct 1998; 18(1): 42 .

7. Dean ACB, Newell JP. Colonoscopy in the differential diagnosis of carcinoma from diverticulitis of the sigmoid colon. $\mathrm{Br} \mathrm{J}$ Surg 1973; 60(8): 633-5.

8. Unal S, Dogan UB, Ozturk Z, Cindoruk M. A randomized prospective trial comparing 45 and $90-\mathrm{ml}$ oral sodium phosphate with $\mathrm{X}$-prep in the preparation of patients for colonoscopy. Acta Gastroenterol Bel 1998; 61(3): 281-4.

9. Girard CM, Rugh KS, Dipalma JA, Brady III CE, Pierson WP. Comparison of Golytely lavage with standard diet/cathartic preparation for double-contrast barium enema. Am J Roentgenol 1984; 142: 1147-9.

10. Afridi SA, Barthel JS, King PD, Pineda JJ, Marshall JB. Prospective, randomized trial comparing a new sodium phosphate-bisacodyl regimen with conventional PEG-ES lavage for outpatient colonoscopy preparation. Gastrointest Endosc 1995; 41(5): 485-9.

11. Warner BA, Dipalma JA. Oral sodium phosphate catharsis: "first do not harm". Am J Gastroenterol 1994; 89(7): $1118-19$.

12. Dipalma JA; Brady III CE, Stewart DL, Karlin DA, Mckinney MK, Clement DJ, Coleman TW, Pierson WP. Comparison of colon cleansing methods in preparation for colonoscopy. Gastroenterology 1984; 86(5): 856-0.

\section{Endereço para correspondência}

Paulo Miki Jr

Disciplina de Proctologia - Departamento de Cirurgia e Anatomia

Faculdade de Medicina de Ribeirão Preto - USP

Campus Universitário

14048-990 - Ribeirão Preto - SP

e-mail -saomarcos@asbyte.com.br 